\title{
Asthma protection with bacteria - science or fiction?
}

\section{Harald Renz}

What is the role of bacterial exposure in asthma protection and prevention? This topic is receiving increasing attention, particularly based on epidemiological findings relating to the 'hygiene hypothesis'. More than a decade ago the observation was made that infants living in a microbial-rich environment show a high degree of protection, particularly against respiratory allergies and allergic sensitisation. ${ }^{1}$ The model situation of the farming environment offers the opportunity to investigate the context of environmental exposures, the effect on immune responses and development of disease phenotypes. That microbes are indeed relevant in triggering the protective immune response was epidemiologically shown initially for exposure to lipopolysaccharide (LPS), a membrane component of Gram-negative bacteria. In that study, an inverse relationship was observed between the natural microbial load (endotoxin load in mattress dust) and the prevalence of asthma, allergic rhinitis and atopic sensitisation. Subsequently, several groups employed models of experimental asthma to study the mechanisms of protection in this regard. ${ }^{2-4}$ These studies provided important information for the context of microbial exposure and asthma protection. First, it was shown that timing of exposure is relevant. If LPS was delivered well before the first contact with the allergen, the immune system was able to mount a clear protective response. In contrast, when LPS was given either simultaneously or even after allergic sensitisation was established, protection was absent, or even in some cases an exacerbation of the inflammatory response was seen. The latter finding is also in line with many clinical observations indicating that acute infections of the upper respiratory tract represent an important trigger for asthma exacerbation. Secondly, dosing is relevant. This finding is not surprising, but further substantiates the epidemiological

Correspondence to Harald Renz, Institute of Laboratory Medicine, Philipps-University Marburg, Baldingerstrasse, D-35043 Marburg, Germany;

renzh@med.uni-marburg.de observations. Thirdly, the route of exposure is relevant. The most consistent findings were observed when the microbial compounds were delivered through the respiratory mucosal tissue.

The next important step forward was the question of whether only LPS would be able to mount such a protective response. Further studies along these lines clearly illustrated that not only Gram-negative but also Gram-positive bacteria were able to provide this protection ${ }^{5-7}$ and this effect is not even restricted to bacterial compounds, since recently it was observed that even the plant product arabinogalactan can induce this protective effect. ${ }^{8}$

Concerning the mucosal aspects, the immune response is initiated by the recognition of microbial compounds by pattern recognition receptors (PRRs). The Toll-like receptor (TLR) family represents one important group of such PRRs which has received a lot of attention recently. TLRs are expressed on a variety of cells, but particularly on airway epithelium, antigen-presenting cells and even cellular subsets of the adaptive immune response in the lung and other organs. It is, therefore, an important question whether activation of TLRs in the airways and the lung would be able to trigger an immune response relevant to asthma protection. This has been tested in a variety of different models with quite consistent outcomes. Activation of TLR2 via synthetic lipopeptides, ${ }^{9}$ stimulation of TLR2/4 heterodimers by peptidoglycans, stimulation of TLR4 via LPS, ${ }^{2}{ }^{3}$ direct activation of TLR3 with poly (I:C) and TLR7 by the compound $\mathrm{R} 848,{ }^{10}$ and activation of TLR9 by DNA extracted from Bordetella pertussis ${ }^{11}$ were all able to trigger the protective immune response. However, the underlying mechanisms show substantial differences. Although in most studies the protection was directly or indirectly linked to the stimulation of a $\mathrm{T}$ helper 1 (Th1) immune response, protection via activation of TLR3 and TLR7 was related to the production of interleukin 12 (IL-12) and IL-10. These data already indicate that there are most probably different immunological pathways associated with and leading to asthma protection. Clearly, the main pathway in this regard is the activation and stimulation of a Th2-counteracting Th1 immune response with high levels of interferon $\gamma$. However, alternative mechanisms via triggering of anti-inflammatory responses and activation of regulatory $\mathrm{T}$ cells must also be considered.

That TLRs are indeed important receptors associated with allergy and asthma protection was again delineated from epidemiological (sub)studies. It was shown that children from a farming environment have increased levels of TLR2, TLR4 and CD14 mRNA, ${ }^{12}$ and the exposure to an increased number of animal species in this environment is also associated with higher TLR expression levels. ${ }^{13}$

In this context, the paper by Nembrini et al in Thorax (see page 755) adds important additional information. ${ }^{14}$ First, the investigators were able to demonstrate that Escherichia coli also delivers this protection. The investigators then went on to demonstrate that TLR4 activation (most probably via LPS) provides an important signal in this regard. This also shows the relevance of mucosal delivery and the interaction with PRRs. It is an important finding that the mechanism of protection is related to the recruitment of $\gamma \delta$-T cells and that neither the Th1 immune response nor regulatory $T$ cells or dendritic cells, nor indeed tumour necrosis factor $\alpha$ was able to overcome the protection or to deliver protective signals. Therefore, this study further underscores that a variety of different mechanisms are able to impact phenotype development in the lung.

From all these observations, several important questions now remain outstanding. Is there any bacterial compound or bacterial strain which does not exhibit such a protective effect when delivered in an appropriate setting and with an appropriate protocol? How important are quantitative exposures over time and what is the duration of the exposure needed to mount a sustainable protective response? How safe is this mode of protection and can it be pharmaceutically industrialised to a level that can be offered as an 'asthma vaccine' to the general population, or to at least a subset with high risks? Answering these and other questions will finally prove the concept which was initiated by the hygiene hypothesis. This line of research has clearly now reached a level of translational sciences and must be tested now in clinical studies. 
Competing interests None

Provenance and peer review Commissioned; not externally peer reviewed.

Published Online First 20 May 2011

Thorax 2011:66:744-745.

doi:10.1136/thx.2011.160853

\section{REFERENCES}

1. Braun-Fahrlander C, Riedler J, Herz U, et al. Environmental exposure to endotoxin and its relation to asthma in school-age children. $N$ Engl $\mathrm{J} \mathrm{Med}$ 2002;347:869-77

2. Blumer N, Herz U, Wegmann M, et al. Prenatal lipopolysaccharide-exposure prevents allergic sensitization and airway inflammation, but not airway responsiveness in a murine model of experimental asthma. Clin Exp Allergy 2005:35:397-402.

3. Gerhold K, Bluemchen K, Franke A, et al. Exposure to endotoxin and allergen in early life and its effect on allergen sensitization in mice. J Allergy Clin Immunol 2003;112:389-96.
4. Eisenbarth SC, Piggott DA, Huleatt JW, et al. Lipopolysaccharide-enhanced, toll-like receptor 4-dependent T helper cell type 2 responses to inhaled antigen. J Exp Med 2002;196:1645-51.

5. Debarry J, Garn H, Hanuszkiewicz A, et al. Acinetobacter Iwoffii and Lactococcus lactis strains isolated from farm cowsheds possess strong allergy-protective properties. J Allergy Clin Immunol 2007:119:1514-21.

6. Blumer N, Sel S, Virna S, et al. Perinatal maternal application of Lactobacillus rhamnosus GG suppresses allergic airway inflammation in mouse offspring. Clin Exp Allergy 2007;37:348-57.

7. Vogel K, Blumer N, Korthals M, et al. Animal shed Bacillus licheniformis spores possess allergy-protective as well as inflammatory properties. J Allergy Clin Immunol 2008;122:307-12, 312.e1-8.

8. Peters $\mathbf{M}$, Kauth $\mathbf{M}$, Scherner 0 , et al Arabinogalactan isolated from cowshed dust extract protects mice from allergic airway inflammation and sensitization. J Allergy Clin Immunol 2010:126:648-56, e1-4.

9. Patel M, Xu D, Kewin P, et al. TLR2 agonist ameliorates established allergic airway inflammation by promoting Th1 response and not via regulatory $T$ cells. J Immunol 2005; 174:7558-63

10. Sel S, Wegmann M, Sel S, et al. Immunomodulatory effects of viral TLR ligands on experimental asthma depend on the additive effects of IL-12 and IL-10. J Immunol 2007;178:7805-13.

11. Kim YS, Kwon KS, Kim DK, et al. Inhibition of murine allergic airway disease by Bordetella pertussis. Immunology 2004;112:624-30.

12. Lauener RP, Birchler T, Adamski J, et al, ALEX study group. Expression of CD14 and Toll-like receptor 2 in farmers' and non-farmers' children. Lancet 2002;360:465-6.

13. Ege MJ, Bieli C, Frei R, et al, Parsifal Study team. Prenatal farm exposure is related to the expression of receptors of the innate immunity and to atopic sensitization in school-age children. J Allergy Clin Immunol 2006;117:817-23.

14. Nembrini C, Sichelstiel A, Kisielow J, et al. Bacterial-induced protection against allergic inflammation through a multicomponent immunregulatory mechanism. Thorax 2011:66:755-63.

\title{
Cardiovascular mechanisms of death in severe COPD exacerbation: time to think and act beyond guidelines
}

\author{
Leonardo M Fabbri, ${ }^{1}$ Bianca Beghé, \\ Alvar Agusti
}

Three important studies on acute exacerbations of chronic obstructive pulmonary disease (ECOPD)have been published in Thorax. Two of them, by Chang et al ${ }^{1}$ (see page 764) and Hoiset et al (see page 775), show the importance of the cardiac biomarkers troponin $\mathrm{T}$ and NT-BNP (Nterminal pro-B-type natriuretic peptide) as strong predictors of the increased risk of death of patients hospitalised because of ECOPD. ${ }^{12}$ The third, by Maclay et $a l^{3}$ (see page 769), provides evidence that patients with stable chronic obstructive

\footnotetext{
${ }^{1}$ Section of Respiratory Diseases, Department of Oncology Haematology and Respiratory Diseases, University of Modena and Reggio Emilia, Policlinico di Modena, Italy; ${ }^{2}$ Thorax Institute, Hospital Clínic, IDIBAPS, Universitat Pompeu Fabra, Centro de Investigación Biomédica en Red de Enfermedades Respiratorias (CIBERES), Barcelona, Spain
}

Correspondence to Leonardo M Fabbri, Section of Respiratory Diseases, Department of Oncology Haematology and Respiratory Diseases, University of Modena and Reggio Emilia, Policlinico di Modena, Italy; leonardo.fabbri@unimore.it pulmonary disease (COPD) have increased circulating platelet-monocyte aggregates-a potential specific pathogenic mechanism of atherosclerosis. These aggregates further increase during exacerbations, suggesting a plausible biological mechanism to explain the increased cardiovascular risk seen in ECOPD. Taken together, these studies confirm the view that ECOPD episodes requiring hospitalisation must be considered very severe events in the natural course of the disease because they are associated with such important outcomes as increased risk of mortality, reduced health status, impaired lung function, muscle weakness, and cardiopulmonary complications. ${ }^{4}$ The studies also suggest that the increased risk of death is often due to acute cardiovascular involvement, and they highlight the limitations of the current definition of ECOPD and the need to move towards a more comprehensive definition, diagnostic approach and treatment.

\section{RISK OF DEATH AND EXACERBATIONS OF} COPD

Several previous studies have clearly established that episodes of ECOPD are a major driver of mortality in this disease, especially during and immediately after the acute event. (The long-term effects are discussed in next paragraph.) For instance, in a cohort of 1016 patients hospitalised because of ECOPD with hypercarbia $\left(\mathrm{PaCO}_{2} \geq 50 \mathrm{~mm}\right.$ $\mathrm{Hg})$, Connors et a ${ }^{5}$ reported that mortality was $11 \%$ in hospital, and 20\%, 33\%, $43 \%$ and $49 \%$, respectively, at 60 days, 180 days, 1 year, and 2 years after discharge. More recently, Chang et $a l^{6}$ reported that mortality during the first 30 days after hospitalisation varied between $2 \%$ and $21 \%$ according to the CURB65 score. CURB65 is a composite index based on confusion, blood urea, respiratory rate, blood pressure and age that was developed to predict mortality risk in community-acquired pneumonia. ${ }^{6}$ Together these studies demonstrate that mortality during and immediately after hospitalisation for ECOPD is remarkably high.

Episodes of ECOPD also affect longterm mortality. This is best illustrated by the study of Soler-Cataluña et al, ${ }^{7}$ who followed a cohort of 304 patients with COPD for 5 years and found that mortality increased in direct proportion to the frequency of ECOPD; that is, patients with frequent episodes (three or more) had the greatest mortality risk (HR 4.13; $95 \%$ CI 1.80 to 9.41 ). In agreement with Connors et al, ${ }^{5}$ Soler-Cataluña et al also found that arterial hypercarbia was an independent marker of poor prognosis (HR 1.07; 95\% CI 1.02 to 1.12). 